\title{
EL MERCADO Y SUS PROYECCIONES EN LA PROPIEDAD EN ROMA ARCAICA
}

\author{
MARKET AND ITS PROJECTIONS INTO OWNERSHIP IN \\ ARCHAIC ROME
}

\section{Carlos Felipe Amunátegui Perelló}

\begin{abstract}
RESUMEN: La propiedad consiste en un derecho de carácter abstracto en el sentido que es susceptible de ejercerse sobre toda clase de bienes con tal que sean, a lo menos, corporales. De esta manera, sujeta a un mismo conjunto de reglas de transacción a una amplia gama de bienes de capital y de consumo, de tal manera que todos quedan sometidos a un régimen de circulación homogéneo. Esta característica, frecuentemente obviada por los juristas al tratar el dominio, permite el desarrollo de una economía de mercado, por lo que tiene una enorme importancia económico-social. Se desarrolló en Roma producto del desarrollo de una economía de mercado en torno al siglo II a.C. y fue heredada por el mundo occidental.
\end{abstract}

Palabras clave: Mercado, propiedad, Roma.

ABSTRACT: Ownership, as a right, has an abstract character, for it can be exercised over a vast set of assets, at least over all corporal goods. It puts under the same transaction rules a large set of assets, without regard to whether they are capital or products, putting them all under the same circulation rules. This feature, sometimes neglected by legal scholars when analyzing ownership, makes possible the development of a market economy and therefore has a large socioeconomic importance. It was developed in Rome during the transformation of its economy into a market one during the 2nd century BC and was inherited by Modern world.

Key words: Market, ownership, Rome.

\section{EXPLICACIÓN GENERAL DEL PROBLEMA}

Dentro del estudio de la historia del dominio, relaciones entre propiedad y mercado no suelen ser enfatizadas con la fuerza que sería deseable. Sin embargo, estas son estrechas y fundamentales, tanto que la concepción actual del dominio es esencial para la construcción de una economía de mercado. En efecto, la propiedad, al extenderse, a lo menos, a todas las cosas corporales, permite tratarlas bajo un sistema uniforme de circulación y comercio, haciendo sus reglas aplicables a un amplio espectro de bienes permitiendo su intercambio económico bajo un marco normativo simplificado y predecible. Así, un trozo de pan y un aeroplano comparten un mismo marco normativo y pueden ser tratados de una misma manera al integrarse en mercados destinados a su circulación económica, puesto que, desde el punto de vista jurídico, se hace abstracción de sus características específicas convirtiéndolos

\footnotetext{
* Profesor de Derecho Romano, Pontificia Universidad Católica de Chile. Dirección postal: Av. Libertador Bernardo O’Higgins 340, Santiago. Correo electrónico: camunate@uc.cl. El presente artículo es parte de los proyectos de investigación Fondecyt Regular 1141231 y Anillos de Investigación Asociativa SOC 1111.
} 
a ambos en objeto del mismo derecho. Esta característica de la propiedad, su abstracción ${ }^{1}$ -que es la que permite el intercambio económico y otorga seguridad en el tráfico jurídico- se encuentra lejos del sentido común o de un desarrollo natural automático desde los instintos de apropiación que los seres humanos compartimos con el conjunto de mamíferos superiores, sino que es fruto de una evolución plurisecular que tiene su origen en la Antigüedad Clásica, específicamente en los dos últimos siglos de la República romana, que es donde la propiedad, como concepto jurídico y económico, se construyó ${ }^{2}$.

La concepción del derecho de propiedad en los distintos sistemas jurídicos modernos no es resultado de un desarrollo autónomo del pensamiento jurídico-económico occidental, sino que es fruto de la recepción de la elaboración jurídica romana realizada en Europa Occidental durante el bajo Medioevo $^{3}$, el cual fue adaptado a las necesidades de la Europa tardomedieval -con mercados en pleno desarrollo- y reelaborado durante la primera parte del siglo XIX durante el movimiento codificador, para un mundo en plena revolución industrial.

Este proyecto de artículo intenta abordar el estudio del surgimiento de la noción de propiedad en el contexto del establecimiento de una economía de mercado en Roma, analizando el origen de la misma y su función económica.

\section{ROMA Y SISTEMAS DE APROPIACIÓN PREVIOS A LA PROPIEDAD}

No todos los sistemas de producción y asignación de recursos -o economías- se fundamentan en la prevalencia del mercado. Siguiendo a Polanyi ${ }^{4}$, podemos clasificarlos en de reciprocidad, distributivos o de intercambio, siendo la economía de mercado una subespe-

\footnotetext{
1 Al respecto de la abstracción de la propiedad, véase CAPOgrossi-Colognessi (2010a) pp. 299-323.

2 En efecto, como ha demostrado abundantemente Capogrossi-Colognesi, el término dominium para abarcar todo el conjunto de bienes corporales comienza a utilizarse durante el último siglo de la República. En el lenguaje jurídico, en tanto, la primera referencia con la que contamos pertenece al jurista Alfeno Varo (s.I a.C.) y se refiere a servidumbres (D.8,3,30). Vid. CApogrossi-Colognesi (1969) pp. 473-488.

3 El régimen jurídico de los bienes durante el alto Medioevo se caracteriza por su fundamental dispersión. En efecto, no existe un régimen unitario capaz de englobar a todos los bienes corporales y otorgarles un tratamiento jurídico uniforme, sino que los distintos usos económicos dan lugar a derechos diferentes. En este sentido, la introducción de la compilación justinianea como base para el estudio del Derecho a contar del siglo XII resultó fundamental a la hora de elaborar un concepto de propiedad. La primera recepción del mismo se encuentra, históricamente, en las Siete Partidas:

P 3, t. XXVIII, L. 1 Señorío es poder que ome ha en su cosa de fazer della, e enella lo que quisiere: segun Dios e segund fuero.

No obstante, suele señalarse que la primera definición de propiedad habría sido obra del postglosador Bartolo de Sassoferrato. Vid. Piccinelli (1980) pp. 42 y ss. Entre los autores más recientes y con bibliografía más abultada que afirman exactamente esto encontramos a RüFNER (2010) pp. 127-142. La definición de Bártolo se encuentra contenida en BARTOLI a Saxoferrato $(1574=2004)$ p. 185 y reza: dominium est ius de re coporali perfecte disponendi, nisi lex prohibeatur.

Sea como fuere, para la elaboración de ambas definiciones se utilizaron dos fragmentos del Corpus Iuris, a saber, C.4,35,21, donde se diferencian las facultades del propietario sobre sus propias cosas, a diferencia de quien actúa por cuenta y riesgo ajeno y D.1.5.4.1, donde Florentino define la libertad. Para más información, véase Amunátegui (2012) pp. 77-120.

4 Polanyi (1976) pp. 289-315.
} 
cie de los sistemas de intercambio específicamente caracterizada por la acción del mercado como fijador de los precios. Así, una economía será propiamente de mercado cuando todos los bienes que participan de ella sean transables en el mismo, sean de capital o de consumo. En este sentido, históricamente han existido diversos sistemas de producción y asignación de recursos en diversas sociedades, siendo el mercado uno de tantos sistemas posibles que ha generado la humanidad. Así, Roma ha pasado por formas económicas muy diversas en su Historia, donde las relaciones entre los hombres y los bienes han mostrado una diversidad correlativa.

En efecto, la sociedad de carácter gentilicio que parece componer el núcleo de la Roma original de los siglos VIII y VII a.C. ${ }^{5}$ no es acorde con una economía donde el mercado jugase un rol central. Las gentes, como unidades clánicas con un territorio, milicia y autoridad política propias ${ }^{6}$, parecen haber controlado gran parte del territorio romano ${ }^{7}$ y haber llevado en su interior una economía más bien distributiva que centrada en el mercado. A la inversa, en sus relaciones intergentilicias el elemento central parece estar constituido por la reciprocidad y el comercio de presentes ${ }^{8}$. El comercio con mercados solo haría su aparición hacia mediados del siglo VII a.C. ${ }^{9}$, con la ascensión al poder de los Tarquinos. En efecto, a contar de la ocupación del poder por reyes etruscos es que se construye un lugar específicamente destinado al comercio -el Forum Boarium $-{ }^{10}$ y, lo que es más importante, se otorga una cierta garantía estatal a los lingotes de bronce marcándolos, lo cual permitirá una primera monetarización de la economía ${ }^{11}$ y la división de la sociedad romana en clases según el censo.

\footnotetext{
5 Así, el área del Lacio durante el período III (c.850-750 a.C.), se encuentra en una suerte revolución social, que comienza con el cierre de la necrópolis del foro y el establecimiento de cabañas en la cima del monte Palatino. Durante este período se observa un progresivo enriquecimiento de los ajuares funerarios que suele identificarse con la formación de las primeras diferencias de clases. Con el enriquecimiento de los mismos pasamos al período IVa (750-650 a.C.), donde aparecen las llamadas tumbas principescas. En estas se aprecian elementos de sorprendente lujo que solo pueden haber sido obtenidos a través del comercio de larga distancia, como objetos de oro y marfil. La evidencia parece respaldar la existencia de una sociedad progresivamente más rica, donde los líderes de pequeños grupos sociales - gentes- manifiestan una creciente diferencia social respecto a los demás.

Véase al respecto, De Martino, Francesco (1997a) pp. 1-26, como también De Martino (1985) pp. 10 y ss.; AlFölDy (1987) pp. 16-22 y con mucho detalle CORNell (1995) pp. 48-56.

Sobre el carácter gentilicio de la misma, ya hemos escrito detalladamente sobre la materia en AMUNÁTEGUI (2009) pp. 11-23.

${ }^{6}$ Véase Bonfante (1963) pp. 7 y ss., como también Bonfante (1916); Arangio-Ruiz (1914); Voci (1953) pp. 101 y ss.; Kaser (1960); Pugliese (1985) pp. 11 y ss. y Franciosi (1995) pp. 88 y ss.

7 Bonfante (1966) pp. 248-9; Diósdi (1970) p. 36; De Martino (1985) p. 15; Stojcevic (1984) p. 1929; Salazar Revuelta (2003) p. 55; Serrao (2006) p. 45.

8 Polanyi (1976) pp. 289-315.

9 Este es el período conocido como IVb orientalizante, caracterizado por la pavimentación del foro, la construcción de cloacas, el reemplazo de las estructuras de madera por otras de ladrillo y otros avances importantes en torno a la constitución del entramado material de la polis. Véanse al respecto Cornell (1995) pp. 81-86 y SMITH (1996) p. 186.

10 SMith (1996) pp .179 y ss.; Ogilvie (1981) p. 33.

${ }^{11}$ Esta información está contenida en la conocida referencia de Plinio el Viejo (Naturalis Historia, 33.43) acerca de la moneda en Roma, que atribuye la creación del aes signatum a Servio Tulio. En todo caso, esta noticia parece provenir de Timeo de Tauromenio, un autor cronológicamente bastante cercano a los hechos, lo que
} 
No obstante, a pesar de introducción de una forma monetaria en la economía romana y del establecimiento de mercados físicos, la configuración de esta no se corresponde con una economía de mercado. En primer término, buena parte de la tierra (el principal capital fijo) seguirá estando en manos de los clanes tal vez hasta el siglo IV a.C., con lo cual queda excluido del mercado uno de sus factores más importantes ${ }^{12}$. Por otro lado, buena parte del territorio conquistado por Roma a sus vecinos se mantiene bajo una nominal titularidad pública (ager publicus) en un régimen de apropiabilidad por ocupación (ager ocupatoribus $)^{13}$ cuya enajenabilidad no parece clara ${ }^{14}$. La progresiva desarticulación de los clanes y la resolución de los conflictos patricio-plebeyos traerá una cierta homogeneidad en las relaciones de apropiación de los bienes, las cuales, a contar del siglo IV serán exclusivamente individuales (quedando atrás otras formas de control grupal de la tierra y otros bienes de capital $)^{15}$. No obstante, todavía no se ha construido un derecho de propiedad que abarque todos los bienes corporales, sino que, lejos de ello, existe una pluralidad de formas jurídicas que implican distintos estatutos para los diversos bienes que las componen. Así, la tierra puede estar bajo estatuto público o privado, con importantes diferencias en cuanto a su apropiabilidad y enajenabilidad, mientras que respecto a los bienes sometidos a un régimen privado existe además una diferencia fundamental entre ciertos bienes de especial importancia económico social cuya circulación es de carácter restringido -las res mancipi- y otros de libre circulación económica que se enajenan a través de la simple entrega ${ }^{16}$. Estas dicotomías entrarán en crisis durante los dos últimos siglos de la República, período en el cual se llevará adelante la privatización de la tierra de toda Italia ${ }^{17}$, por una parte, y la homogenización de los derechos y formas de circulación de los bienes de capital y productos. Esto último se realiza a través de la creación de la llamada propiedad bonitaria mediante la cual se validan -a través de la acción publiciana- las enajenaciones no formales realizadas sobre bienes mancipi.

En definitiva, hacia fines de la República, al crearse el concepto de dominio como un derecho omnicomprensivo aplicable a la generalidad de los bienes corporales, se crean los mecanismos jurídicos necesarios para someter a todos los bienes de producción al mercado, incluida buena parte de la mano de obra, que queda dentro de un mercado no del trabajo

le otorga cierta verosimilitud (SERRAO (1981) p. 75). En todo caso, la mejor confirmación de la narración de Plinio está en el hecho de haberse encontrado en Thesmophoros ejemplares similares a los descritos. Vid. DE Martino (1985) pp. 67-68.

12 En efecto, hay cierto acuerdo en que la mancipatio original no parece estar diseñada para la tierra MOMMSEN (1902) p. 438; Capogrossi-Colognesi (1981) p. 139; Behrends (1982) p. 50.

13 Al respecto, véase el clásico WeBER (1982) pp. 89-93.

14 Respecto a la particular situación del ager publicus, tal vez la tesis más convincente es aquella de CAPOGROSSIColognesi que estima que hasta la dictación de las leyes Liciniae-Sextiae (367 a.C.) el ager publicus solo podía ser ocupado por las gentes. CAPOGrossi-COLOGNeSI (1981) pp. 3-45

15 Véase Serrao (2006) pp. 273-276.

16 Gallo (2004=1958) p. 58; Arangio-Ruiz (1987=1956) pp. 18 y ss; Capogrossi-Colognesi (1969) p 132; Westbrook (1989) p. 207; Varela (2007) pp. 4 y ss.

17 Capogrossi-Colognesi (2010b) pp. 129-142. 
sino de trabajadores bajo el sistema de esclavitud ${ }^{18}$, aunque también parece existir un importante elemento libre en la mano de obra ${ }^{19}$.

\section{PROPIEDAD Y MERCADO}

El proceso de desarrollo del concepto de propiedad en Roma parece compatible con el establecimiento durante la República tardía de una economía de mercado, aunque desde mediados de los setenta del siglo XX esto parece difícil de sostener. En efecto, desde la publicación del ya clásico trabajo de Finley, Ancient Economy, se ha consolidado la idea de una economía romana donde los mercados jugaban un rol muy secundario ${ }^{20}$ y de escasa monetarización ${ }^{21}$, con la salvedad de ciertos núcleos urbanos especialmente importantes. En efecto, la ortodoxia actual sobre la economía romana tiende a concebirla desde un punto de vista que ha sido llamado "primitivista", toda vez que minimiza el rol de los mercados en la fijación de precios y en el desenvolvimiento general de la economía romana.

No obstante, esta visión ha sido fuertemente debatida en la última década en cada uno de sus aspectos, desde su monetarización ${ }^{22}$, hasta el rol de los mercados ${ }^{23}$ y la posibilidad de crecimiento económico en la Antigüedad ${ }^{24}$. En este sentido, llama poderosamente la atención la ausencia del punto de vista jurídico de la misma, toda vez que el Derecho romano parece ser uno de los sistemas jurídicos más fuertemente orientados a la prevalencia de una economía de mercado de cuantos han existido. En efecto, aunque no hayan contado con una teorización sobre los mercados, el sistema jurídico como tal buscaba la preservación de las relaciones económicas ${ }^{25}$. Así lo declara Ulpiano expresamente:

D.1,3,41 Ulpianus libro II institutionum. Totum autem ius constitit aut in adquirendo aut in conseruando aut in minuendo: aut enim hoc agitur quemadmodum quid cuiusque fiat, aut quemadmodum quis rem uel ius suum conseruet, aut quomodo alienet aut amittat.

${ }_{18}$ Si bien la esclavitud es bastante anterior al período en estudio, toda vez que parece haber sido introducida ya durante la monarquía etrusca: Franciosi (1959) p. 375; Franciosi (1992) p. 206; De Martino (1997b) v. III, pp. 82-83; De Martino (1997c) pp. 27-57.

19 En todo caso, recientemente el estudio del trabajo libre en Roma tardorrepublicana e imperial parece cobrar mayor importancia e incluso se plantea la posible existencia de un verdadero mercado de trabajo en Roma. En este sentido resultan importantes: HarRIS (2011a) 1.546-578 y KeHOE (2012) pp. 114-131. La visión más extrema al respecto: Temin (2013) pp. 114-138.

${ }^{20}$ No obstante, debe reconocerse que el mismo Finley es menos drástico en sus afirmaciones que sus seguidores. En efecto, Finley no descarta por completo la acción del mercado ni intenta mostrar una economía donde ellos directamente no operasen, sino que simplemente los estima, en la mayor parte de los casos, secundarios. Así, por ejemplo, señala que los grandes terratenientes romanos producirían cosechas exclusivamente orientadas a los mercados (chash-crops), aunque destaca la preponderancia absoluta del modelo productivo del campesino autosuficiente. Vid. Finley (1999) pp. 95-122. En el mismo sentido: Saller (2002) pp. 251-269.

${ }^{21}$ En este sentido resulta fundamental CraWford (1970) pp. 40-48.

22 En favor de una mayor monetarización de la economía: Greene (1990) pp. 45-66 y Von Reden (2012) pp. 266-285. A propósito de la presencia de dinero no metálico, sino incluso crediticio, en la economía romana: HarRis (2011b) 1.2557-2936. Postulando la existencia de un mercado financiero para Roma: Temin (2013) pp. 157-191

${ }^{23}$ Hopkins, Keith (2002) pp. 190-232.

${ }^{24}$ Temin, Peter (2013) pp. 193-261.

25 Véase al respecto: ViVENZA (2012) pp. 25-44. 
El desarrollo de la compraventa obligacional, por ejemplo, revela un pensamiento económico sofisticado ${ }^{26}$ especialmente ya que no solo es apta para la venta a crédito -toda vez que el precio no debe pagarse necesariamente en el acto y esto no acarrea efectos sobre la transferencia dominical-, sino también para constituir mercados sobre bienes futuros, pues permite la venta de cosas que no existen. Otro tanto podemos decir del hecho que la condemnatio en el procedimiento formulario deba ser siempre en dinero y que no exista una condena en especie ${ }^{27}$, puesto que implica que todas las cosas tienen un valor de mercado, toda vez que es a través del mismo que generalmente se determinaba su monto. Es más, las diversas sutilezas respecto al valor de mercado según el momento en que se contrajo la obligación o de la sentencia, reflejadas en los tiempos verbales usados en la fórmula, implican una amplia variabilidad de los precios en el mercado, una característica central de toda economía de mercado ${ }^{28}$.

En este contexto, el desarrollo del concepto de dominio en Roma tardorrepublicana parece otro elemento más que apunta directamente a la existencia de una economía de mercado, fuertemente monetarizada y con todos sus bienes, sean de capital o de consumo, sometidos al libre juego del mismo.

\section{BIBLIOGRAFÍA CITADA}

Alföldy, Géza (1987): Historia Social de Roma (Alianza, Madrid).

Amunátegui Perelló, Carlos Felipe (2009): "Roma, confederación de gentes”, Studi in onore di Antonino Metro (Milano, Giuffrè) pp. 11-23.

Amunátegui Perelló, Carlos Felipe (2012): "Las relaciones de vecindad y la teoría de las inmisiones", Revista de Derecho (Valparaíso) No 38: pp. 77-120.

Arangio-Ruiz, Vincezo (1914): Le genti e le città (Università di Messina).

ARANGIO-RuIZ, Vincenzo (1987=1956): La compravendita in diritto romano (Jovene, Napoli).

BARTOLI a Saxoferrato $(1574=2004)$ : In primam digesti novi partem commentaria (Augustam

Taurinorum, Nicolaum Belvilaquam = Frankfurt am Main, A.J.B. Sirks).

BeHrends, Okko (1982): "La mancipatio nelle XII Tavole", IURA XXXIII: pp. 46-103.

Bonfante, Pietro (1916): La gens e la familia, scritti giuridici, famiglia e successione (Unione Tipografico, Torino).

Bonfante, Pietro (1963): Corso di diritto romano, Diritto di famiglia (Giuffrè, Milano).

Bonfante, Pietro (1966): Corso di diritto romano, La proprietà (Giuffrè, Milano) v. II, p. I.

CAPOgrossi-Colognesi (1969): Luigi, La struttura della propietà e la formulazione dei iura prediorum nell'età repubblicana (Giuffrè, Milano).

Capogrossi Colognesi, Luigi (1981): La terra in Roma antica (La Sapienza, Roma).

\footnotetext{
26 Sobre el problema, véase: Melillo (2000) pp. 43-75.

27 G. 4.484 .48 Omnium autem formularum, quae condemnationem habent, ad pecuniariam aestimationem condemnatio concepta est. itaque et si corpus aliquod petamus, uelut fundum, hominem, uestem, <aurum>, argentum, iudex non ipsam rem condemnat <e>um, cum quo actum est, sicut olim fieri solebat, <sed> aestimata re pecuniam eum condemnat.

28 En efecto, una característica esencial de los precios en las economías de mercado es que varían. Vid.: TEMIN (2013) pp. 58-59.
} 
Capogrossi-Colognessi, Luigi (2010a): "Das Eigentum in Rom vom Ende der patriarchalischen Ordnung bis zur Blütezeit der Sklavereiordnung”, Scritti scelti (Jovene, Napoli) pp. 299-323.

CAPOgrossi-Colognesi, Luigi (2010b): “Alcuni aspetti dell'organizzazione fondiaria romana nella tarda Repubblica e nel Principato", Scritti scelti (Jovene, Napoli) pp. 129-142.

Crawford, Michael (1970): "Money and Exchange in the Roman World", Journal Roman Studies 60: pp. 40-48.

Cornell, Tim J. (1995): The Beginnings of Rome (Routledge, New York).

De Martino, Francesco (1985): Historia económica de la Roma Antigua (Akal, 1985, Mardid), Tomo I.

De Martino, Francesco (1997a): "La gens, lo stato e le classi in Roma antica", Diritto, economia e società nel mondo romano (Jovene, Napoli) pp. 25-49.

De Martino, Francesco (1997b): Clienti e condizioni materiali in Roma arcaica in Diritto economia e società nel mondo romano (Jovene, Napoli) v. III: pp. 53-83.

De Martino, Francesco (1997c): Intorno all'origine della schiavitù a Roma in Diritto economia e società nel mondo romano (Jovene, Napoli) v. III: pp. 27-57.

DiósDI, György (1970): Ownership in Ancient and Preclassical Roman Law (Akadémiai Kiadó, Budapest).

Finley, Moses I (1999): Ancient Economy (California, London).

Franciosi, Gennaro (1959): "Res mancipi e res nec mancipi”, Labeo 5 3: pp. 370-386.

Franciosi, Gennaro (1992): Famiglia e persone in Roma antica (Giappichelli, Torino).

FrancIOSI, Gennaro (1995): "Gentilicium ius in desuetudinem abiise Ricerche sulla organizzazione gentilizia romana III” (Jovene, Napoli) pp. 85-97.

Gallo, Filippo (2004=1958): "Studi sulla distinzione fra res mancipi e res nec mancipi" Rivista di Diritto Romano, NoIV. Studi sul transferimento della proprietà in diritto romano (Torino) pp. 1-121.

Greene, Kevin (1990): The Archaeology of the Roman Economy (California, Berkeley).

Harris, William V. (2011a): "Poverty and destitution in the Roman Empire", Rome's imperial economy (Oxford, New York, Kindle ed.).

Harris, William V. (2011b): "A Revisionist View on Roman Money", Rome's imperial economy (Oxford, New York, Kindle ed.).

Hopkins, Keith (2002): "Rome, Taxes, Rents and Trade" Schiedel, Walter (Ed.) The Ancient Economy (Routledge, New York) pp. 190-231.

Kaser, Max (1960): "La famiglia romana arcaica", Conferenze romanistiche (Milano, Dott. A. Giuffré) pp. 41-62.

Kehoe, Denis (2012): “Contract labor" Schiedel, Walter (Ed.) Roman Economy (Cambridge, New York) pp. 114-130.

Melillo, Generoso (2000): Categorie economiche nei giuristi romani (Jovene, Napoli).

Mommsen, Theodor (1902): "Mancipium, manceps, praes", praedium in Zeitung der Savigny Stiftung 23: pp. 438-441.

Ogilvie, Robert M. (1981): Roma Antigua y los etruscos (Taurus, Madrid).

Piccinelli, Ferdinando (1980): Studi e ricerche intorno alla definizione dominium est ius utendi et abutendi re sua quatenus iuris ratio pattur (Napoli, Jovene). 
Amunátegui Perelló, Carlos Felipe "El mercado y sus proyecciones en la propiedad en Roma arcaica"

POlanyI, Karl (1976): "La economía como actividad institucionalizada" Polanyi, Karl, Arensberg, Conrad y Person, Harry, Comercio y Mercados en los Imperios Antiguos (Labor Universitaria, Barcelona) pp. 289-315.

Pugliese, Giovanni (1985): Aperçu historique de la famille romaine, Scritti giuridici scelti (Napoli, 1985, Jovene Editore), v III.

Rüfner, Thomas (2010): The Roman Concept of Ownership and the Medieval Doctrine of Dominium Utile in The Creation of Ius Commune. From Casus to Regula, Cairns, John W. y Du Plessis, Paul J. (eds.) (Edinburgh University Press, Edinburgo) pp. 127-142.

Salazar Revuelta, María (2003): Evolución histórico-jurídica del condominio en el Derecho romano (Universidad de Jaén, Jaén).

Saller, Richard (2002): "Framing the debate over growth in the Ancient Economy" Schiedel, Walter (Ed.) The Ancient Economy (Routledge, New York) pp. 223-238.

SERraO, Feliciano (1981): "Lotte per la terra e per la casa a Roma" Legge e società nella repubblica romana (a cura di Feliciano Serrao) (Jovene, Napoli) v. I: pp. 51-180.

SerraO, Feliciano (2006): Diritto privato economia e società nella storia di Roma (Jovene, Napoli), v.1.

SMITH, Christopher John (1996): Early Rome and Latium (Clarendon Press, Oxford).

STOJCEVIC, Dragomir (1984): "Propietà sociale propietà feudale e dominium" Sritti in onore di Antonio Guarino (Jovene, Napoli) v. II: pp. 1.927-1.939.

Temin, Peter (2013): The Roman Market Economy (Princeton, Princeton).

Varela Gil, Carlos (2007): "El origen de la mancipatio: de medio de publicidad dominical a modo de adquirir la propiedad”, Revista General de Derecho Romano, No 9: pp. 1-14.

Vivenza, Gloria (2012): "Roman Economic Thought” Schiedel, Walter (Ed.) Roman Economy (Cambridge, New York) pp. 25-44.

VocI, Pascuale (1953): "Esame delle tesi del Bonfante su la Famiglia Romana Arcaica" Studi in onore di Arangio-Ruiz (Napoli, 1953, Jovene Editore) Tomo I: pp. 101-146.

Von Reden, Sitta (2012): "Money and Finance" in Schiedel, Walter (Ed.) Roman Economy (Cambridge, 2012, New York) pp. 266-286.

WeBER, Max (1982): Historia agraria romana (Akal, Madrid).

Westbrook, Raymond (1989): Restrictions on Alienation in Early Law in Peter Birks (editor), New Perspectives in the Roman Law of Property. Essays for Barry Nicholas (Clarendon, Oxford) pp. 207-227. 